\title{
Screening Test of Greenhouse Seeding Exercise Matrix for Tissue Culture Seeding of Dendrobium Officinale Kimura et Migo
}

\author{
Yuan Zhou ${ }^{1}$, Shaojun Huang ${ }^{1}$, Jianfeng Huang ${ }^{2}$, Yun Liu ${ }^{1}$, Jieqiong Zeng ${ }^{1}$, Xianling Zeng ${ }^{1}$, Wen Huang ${ }^{1}$, and Limei Ou ${ }^{1}$ \\ ${ }^{1}$ Guangxi Agriculture Vocational College, 530007 Nanning Guangxi, China \\ ${ }^{2}$ Extension Station of Guangxi Agricultural Mechanization Technology, 530007 Nanning Guangxi, China
}

\begin{abstract}
The Dendrobium officinale Kimura et Migo has a high demand on planting matrix, while its tissue culture seeding has much more demands on planting matrix.To find out a seeding exercise matrix to enhance the survival rate of tissue culture seeding of Dendrobium officinale Kimura et Migo more efficiently, this article carries out a screening test of greenhouse seeding exercise matrix material for tissue culture seeding of Dendrobium officinale Kimura et Migo. The test adopts full random test design, mainly for screening test of five matrix materials, namely pine bark, camphor tree bark, fern root, peanut shell and longan bark. Compare the impact of prepared seeding exercise matrix on the survival rate and growth trend (including plant height, growth rate and bud growth rate). The test result shows that: The seeding exercise matrix prepared by fern root is the most efficient, and the survival rate, plant height, growth rate and bud growth rate have achieved $100 \%$, $4.5 \mathrm{~cm}, 43.67 \%$ and $54.33 \%$ respectively. The main reason may be that the seeding exercise matrix $\mathrm{C}$ prepared by fern root is fairly loose and has a great water permeability, which is conducive to the growth of Dendrobium officinale Kimura et Migo.
\end{abstract}

Keywords. Dendrobium officinale Kimura et Migo; tissue culture seeding; seeding exercise matrix; screening test

Dendrobium officinale Kimura et Migo belongs to Dendrobium Sw., Orchidaceae. According to Chinese Pharmacy Dictionary, it "nourishes gas-liquid of lung and stomach, making the gas-liquid vigorous and kidney water regenerative", and is good at promoting the secretion of saliva, nourishing yin and treating the yin deficiency, depletion of fluid and other syndromes. It also can strengthen the spleen and stomach, enhance physical fitness, protect the liver and gallbladder, strengthen the muscle, lower the fat, reduce the blood sugar, inhibit the tumor, brighten the eyes, nourish the skin, prolong the life, etc. ${ }^{[1,2,3]}$ In addition, it is mentioned in the Inner Canon of Huangdi that "An excellent doctor will treat the disease to occur instead of having occurred." ${ }^{[4]}$.

As known from above, our human beings had applied the Dendrobium officinale Kimura et Migo to prevent the disease and achieve yin-yang coordination to realize the goal of good health and long-life. In addition, the modern medical research has shown that the Dendrobium officinale Kimura et Migo has abundant Dendrobium polysaccharide component, with the effects like improving immunity, strengthening memory, preventing and fighting the cancer, and prohibiting the tarombokinesis. So, with the constant development of living standard, the people attach more importance to health maintenance.
The Dendrobium officinale Kimura et Migo is not only for medical use, but also used by common people as the first choice of daily drinking, cuisine and appreciation. Furthermore, the market demand is increasing year by year. Currently, the wild resources are close to be depleted and cannot meet the market demand.

The Dendrobium officinale Kimura et Migo belongs to perennial herbage, living by intergrowth. In the current horizontal distribution, it is concentrated on Qinling Mountains and the Yangtze River Basin, gradually reduced from the south provincial area to the north ${ }^{[5]}$. In the current vertical distribution, it is distributed in the altitude of 100-3500 meters. The domestic wild Dendro-bium officinale Kimura et Migo is mainly distributed in Guangxi, Zhejiang, Yunnan, Guizhou and other places ${ }^{[7]}$. Currently, it is usually cultivated in Guangxi, Guangdong, Jiangxi, Anhui, Fujian and other places ${ }^{[8]}$. The Dendrobium officinale Kimura et Migo has a high demand on growth environment, and mostly intergrow on the surface of valley, cliff or bark, where there are many bryophytes in wet and half-shade environment. The range of proper temperature for its growth is $18-30^{\circ} \mathrm{C}$, and $16-21^{\circ} \mathrm{C}$ for growth period, $10-13^{\circ} \mathrm{C}$ for evening temperature. The optimal temperature difference is kept within $10-15^{\circ} \mathrm{C}$. The temperature over $30^{\circ} \mathrm{C}$ has little impact on the 
growth of Dendrobium officinale Kimura et Migo, but the temperature below $10^{\circ} \mathrm{C}$ is not desirable where the seedings are prone to suffer from cold. The root of Dendrobium officinale Kimura et Migo belongs to endogenetic "orchidaceae mycorhiza" without the need to use the root system to absorb the nutrients from the soil to maintain its growth. In addition, its aerial root can only absorb little nutrients from the air and rainfall, with most of nutrients mainly absorbed from dissolving the surrounding residual organisms by mycelium in symbolic relation with its root ${ }^{[8,9,10]}$. So the Dendrobium officinale Kimura et Migo has a high demand on planting matrix to be conducive to the growth of its mycorhiza.

The Dendrobium officinale Kimura et Migo has been ranked as the national key protected 2-class medicine, while its reproduction rate in natural condition is extremely low and the wild resources are depleted day by day due to long-term and unreasonable exploitation. The domestic Dendrobium officinale Kimura et Migo is mainly distributed in Zhejiang, Guangxi, Hunan, Yunnan, Guizhou and other places, and is a famous regional medicine, precious medicine and important ethnic medicine. At the beginning of 2010, Guangxi greatly supported the Chinese herbal medicine planting industry, cultivation area of medicine in Guangxi, including the Dendrobium officinale Kimura et Migo, is increasing day by day, with the market demand exceeding the supply ${ }^{[6]}$. Guangxi becomes the main production area of Dendrobium officinale Kimura et Migo, and the Rong County, Yulin City, Guangxi has been reputed as "Home to Dendrobium officinale Kimura et Migo in Guangxi". Currently, using the tissue culture method to rapidly reproduce the tissue culture seeding has been the efficient way of settling the seeding problems. The growth of Dendrobium officinale Kimura et Migo has a strict demand on moisture, temperature and other factors. Many planters and even farmers can use the greenhouse technology to control, but the tissue culture seeding will have a low survival rate of transplantation if not going through seeding exercise before planted in the greenhouse. So it is currently the most important to settle the problems of low survival rate of transplantation for tissue culture seeding. For general farmer planters, the current tissue culture seeding technology has a high planting and technical demand, with lower survival rate, which means the tissue culture seeding is hard to be planted for the farmers. Many farmers mainly purchase the acclimated seedlings which has gone through the process of seeding exercise, and this will definitely increase their costs. To promote the plantation of Dendrobium officinale Kimura et Migo for the farmers, the priority is to settle the problem of seeding exercise. Therefore, the research group of this test, in consideration of Guangxi, carried out a screening test of greenhouse seeding exercise matrix material for tissue culture seeding of Dendrobium officinale Kimura et Migo, for the purpose of using the accessories with local Guangxi features and screening the seeding exercise matrix capable of enhancing the survival rate of tissue culture seeding of Dendrobium officinale Kimura et Migo to provide an advantage condition of planting the Dendrobium officinale Kimura et Migo for many Guang- xi planters, and let the farmers get higher benefits of economy.

\section{Material and method}

\subsection{Test material}

Test-use tissue culture seeding is purchased from Guangxi Medicinal Botanical Garden Chinese Medicine Seeding Co., Ltd.. This batch of tissue culture seedings were formed by tissue culture in 2013 (subject to sound seeding treatment). Cultivation matrices of pine bark, camphor tree bark, fern root, peanut shell and longan bark are all cultivated from local Guangxi. This test uses seeding exercise bed, with length, width and height respectively $100 \mathrm{~cm}, 30 \mathrm{~cm}$ and $120 \mathrm{~cm}$.

\subsection{Test design}

From March-June in 2013, use the full random test design and set 5 treatments in a potting manner in planting greenhouse (constructed according to general farmer construction specification in Guangxi) of Guangxi Agricultural Vocational College. Each test unit plants a cluster of tissue culture seeding ( 3 for each cluster). Each treatment (matrix) in the matrix screening test will set 3 repetition groups, but not including control group.

\subsubsection{Test process}

Tissue culture seeding treatment: Take the Dendrobium officinale Kimura et Migo seeding out of the tissue culture bottle, use the water to slightly wash the culture medium on the root of seeding. Be careful not to damage the root system and leaves when washing. Then put into the prepared chlorothalonil to be immersed for 30-60s, and finally take out the seeding to be spared at ventilated place. This test will select plant height over $3-4 \mathrm{~cm}$, and tissue culture seeding of Dendrobium officinale Kimura et Migo with over 3 leaves, 4 root systems and dark green stems and leaves.

Treatment of seeding exercise matrix: seeding exercise matrix A: $60 \%$ Pine sawdust $+40 \%$ pine bark; seeding exercise matrix B: $60 \%$ Pine sawdust $+40 \%$ camphor bark; seeding exercise matrix C: $60 \%$ Pine sawdust $+40 \%$ fern root; seeding exercise matrix D: $60 \%$ Pine sawdust $+40 \%$ peanut shell; seeding exercise matrix E: $60 \%$ Pine sawdust $+40 \%$ longan bark; cultivation accessories shall undergone the high pressure and high temperature steam sterilizing $\left(0.15 \mathrm{MP}, 121^{\circ} \mathrm{C}, 1 \mathrm{~h}\right)$. Prepared matrix shall be added with $30 \mathrm{~g}$ of Qingfeishen flower fertilizer (produced by Zhongshan Xingnong Agriculture Service Co., Ltd.), and respectively put in the $1 / 500$ chlorothalonil aqueous solution to be immersed for 7 days. Then take it out to a transparent plastic box until it has no water dropped therefrom, and for storage.

Bed position: The treated seeding exercise matrix will be planted 3 boxes for each matrix, and respectively put into 18 transparent plastic box ( specification is $10 \mathrm{~cm}$ in length, $10 \mathrm{~cm}$ in width, $6 \mathrm{~cm}$ in height). Its volume accounts for $2 / 3$ of that for transparent plastic box. The 
bottom will be added by the pine park going through the sterilization of mineral chameleon $(1-2 \mathrm{~cm})$, so as to increase the breathability for matrix to be planted. Each box will be planted by 1 cluster of seedings of Dendrobium officinale Kimura et Migo (3 plants constitute a cluster), and marked with labels. Randomly put all of the test units to the seed bed.

Management of cultivation: Timely complement the moisture everyday, as the Dendrobium officinale Kimura et Migo has a high demand on water. When watering, we shall use the atomized water and have a strick control on water volume to keep the matrix wet without water collection. Transplanted for half a month, it shall be watered by nutritive medium once for every 15 days (based on wet leaf and waterdrop appearing), Nutritive media with different concentration (This test uses Huaduoduo No. 1) are varied for growth of transplanted seedings. Timely ventilate, weed to prevent plant diseases and insect pests.

\subsubsection{Test item and method}

Calculation of survival rate: After completing the test, record the number of plants that survived for each test unit, and calculate the obtained data. Calculation formula: Survival rate $(\%)=($ After completing the test, the number of plants of tissue culture seeding that survived for each test/ total plants of cultivated tissue culture seeding for this test unit) $\times 100 \%$.

Growth situation record: From the date of cultivation, successively investigate the growth situation among each seeding exercise matrix (plant height and bud growth number), and recored the data.

Calculation of growth rate: After completing the test, record the number of each plant height for each test unit, and calculate the obtained data. Calculation formula: Growth rate $(\%)=($ After completing the test, the average plant height of tissue culture seeding increased for each test unit/The average plant height of tissue culture seeding for each test unit, before the test) $\times 100 \%$.

Calculation of bud growth rate: After completing the test, record the number of increased buds of each plant for each test unit, and calculate the obtained data. Calculation formula: Bud growth rate $(\%)=$ (After completing the test, the average new buds of tissue culture seeding increased for each test unit/The total buds of tissue culture seeding for each test unit, before the test) $\times 100 \%$.

\section{Result and analysis}

\subsection{Impact of different seeding exercise matrix on survival rate}

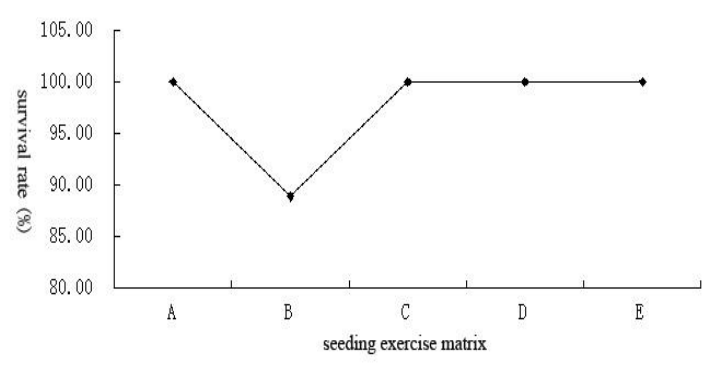

Figure 1. Impact of 5 different seeding exercise matrix on seeding exercise.

As shown in Figure 1, the survival rates of Different for 5 different seeding exercise matrix are high. In the survival rates for 5 seeding exercise treated by matrix, only the survival rate of seeding exercise matrix $\mathrm{B}$ is lower than that for other seeding exercise matrix. After variance analysis, the efficiency difference of survival rate of five seeding exercise matrix is not so obvious.

\subsection{Impact of different seeding exercise matrix on growth situation}

\subsubsection{Impact of different seeding exercise matrix on plant height}

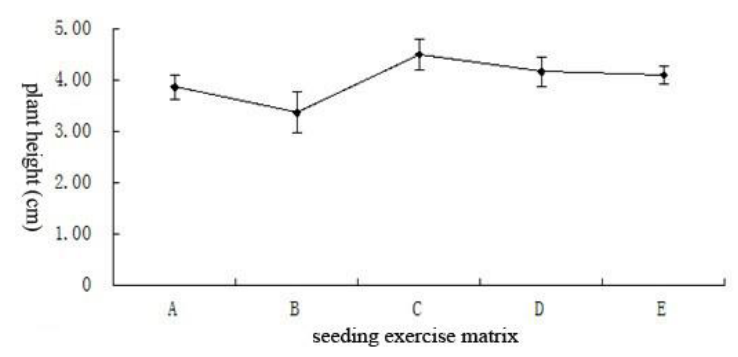

Figure 2. Impact of different seeding exercise matrix on plant height.

As shown in Figure 2, in the same conditions, the plant height of seedings are varied after treatment by different seeding exercise matrix. In 5 Figure seeding exercise matrix, the impact of seeding exercise matrix A and B, compared to the other 4 seeding exercise matrix, on the plant height of seedings is lower. Seeding exercise matrix $\mathrm{C}$ treatment has the largest impact on the plant height of seedings; successively followed by seeding exercise matrix D and seeding exercise matrix $C$. After variance analysis, the efficiency difference of plant height of five seeding exercise matrix is obvious. Further multiple comparison is required (This test use SSR method for all the multiple comparison methods, and the Table comparison use all the method of step table. Multiple comparison result is shown in Table 1:

Table 1. Difference among differently treated plant heights.

\begin{tabular}{llllll}
\hline \multirow{2}{*}{ Treatment (seeding exercise matrix) } & \multirow{2}{*}{ Average plant height } & \multicolumn{4}{l}{ Difference } \\
\cline { 4 - 6 } & & $4.5-$ & $4.17-$ & $4.1-$ & $3.87-$ \\
\hline $\mathrm{C}$ & 4.5 & & & & \\
$\mathrm{D}$ & 4.17 & 0.33 & & & \\
$\mathrm{E}$ & 4.1 & 0.4 & 0.07 & & \\
$\mathrm{~A}$ & 3.87 & 0.63 & 0.3 & 0.23 & \\
$\mathrm{~B}$ & 3.37 & 1.13 & 0.8 & 0.73 & 0.5 \\
\hline
\end{tabular}


As shown in Table 1, the plant height of seeding exercise matrix $\mathrm{C}$ and the impact of four treatment methods of seeding exercise matrix $\mathrm{D}$, seeding exercise matrix $\mathrm{E}$, seeding exercise matrix A and seeding exercise matrix $B$ on plant height is not so obvious.

\subsubsection{Impact of different seeding exercise matrix on growth rate}

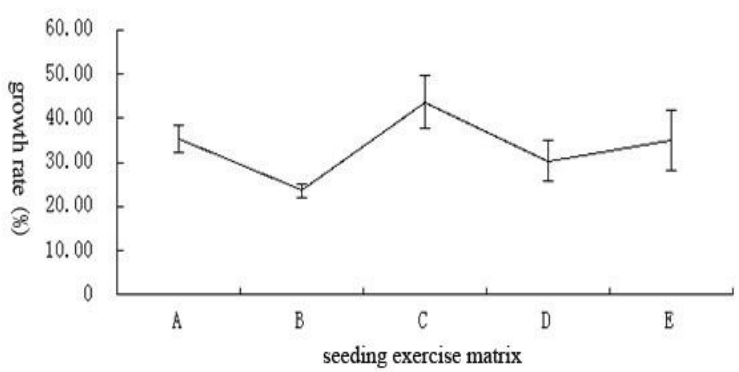

Figure 3. Impact of 5 different seeding exercise matrix on growth rate of Dendrobium officinale Kimura et Migo.

As shown in Figure 3, the bud growth rates for 5 different seeding exercise matrix are obviously different. In comparison of the growth rates of the seeding exercise treated by 5 matrix, the seeding exercise matrix $\mathrm{C}$ is treated well, followed by seeding exercise matrix $\mathrm{D}$, seeding exercise matrix E and seeding exercise matrix A. The seeding exercise matrix B is least affected. After variance analysis, the impact of different seeding exercise matrix on bud growth rate is obvious. Further multiple comparison Multiple comparison result is shown in Table 2:

Table 2. Difference among differently treated growth rate.

\begin{tabular}{|c|c|c|c|c|c|}
\hline \multirow{2}{*}{$\begin{array}{l}\text { Treatment } \\
\text { (seeding exer- } \\
\text { cise matrix) }\end{array}$} & \multirow{2}{*}{$\begin{array}{l}\text { Growth } \\
\text { rate }\end{array}$} & \multicolumn{4}{|c|}{ Difference } \\
\hline & & $43.67-$ & $35.33-$ & $35-$ & $30.33-$ \\
\hline $\mathrm{C}$ & 43.67 & & & & \\
\hline A & 35.33 & 8.34 & & & \\
\hline E & 35 & 8.67 & 0.33 & & \\
\hline D & 30.33 & 13.34 & 5 & 4.67 & \\
\hline B & 23.67 & 20 & 11.66 & 11.33 & 6.66 \\
\hline
\end{tabular}

As shown in Table 2, the plant height of seeding exercise matrix $\mathrm{C}$ and the impact of four treatment methods of seeding exercise matrix $\mathrm{D}$, seeding exercise matrix $\mathrm{E}$, seeding exercise matrix A and seeding exercise matrix B on plant height is not so obvious.

\subsubsection{Impact of different seeding exercise matrix on bud growth rate}

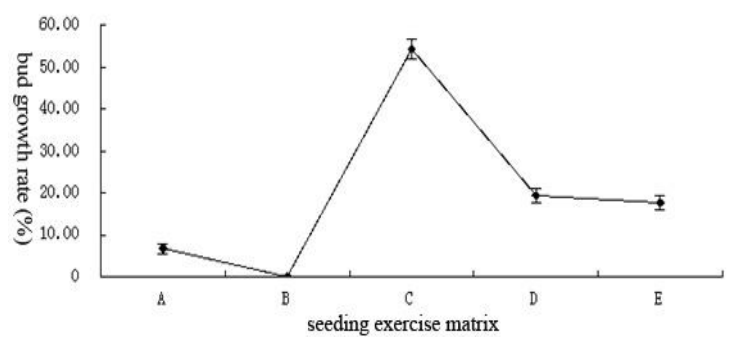

Figure 4. Impact of different seeding exercise matrix on bud growth rate.
As shown in Figure 4, the bud growth rates for 5 different seeding exercise matrix are obviously differnet. In comparison of the bud growth rates of the seeding exercise treated by 5 matrix, the seeding exercise matrix $\mathrm{C}$ is treated well, followed by seeding exercise matrix $\mathrm{D}$, seeding exercise matrix $\mathrm{E}$ and seeding exercise matrix A. The seeding exercise matrix $\mathrm{B}$ is almost not affected. After variance analysis, the impact of different seeding exercise matrix on bud growth rate is obvious. Further multiple comparison Multiple comparison result is shown in Table 3:

Table 3. Difference among differently treated bud growth rate.

\begin{tabular}{llllll}
\hline Treatment & Bud & \multicolumn{2}{l}{ Difference } & & \\
\cline { 5 - 6 } $\begin{array}{l}\text { seeding exer- } \\
\text { cise matrix) }\end{array}$ & $\begin{array}{l}\text { growth } \\
\text { rate }\end{array}$ & $54.33-$ & $19.33-$ & $17.67-$ & $6.67-$ \\
\hline C & 54.33 & & & & \\
D & 19.33 & 30.67 & & & \\
E & 17.67 & 32.33 & 15.33 & & \\
A & 6.67 & 43.33 & 26.33 & 11 & \\
B & 0 & 50 & 33 & 17.67 & 6.67 \\
\hline
\end{tabular}

As shown in Table 3, the plant height of seeding exercise matrix $\mathrm{C}$ and the impact of four treatment methods of seeding exercise matrix $\mathrm{D}$, seeding exercise matrix E, seeding exercise matrix A and seeding exercise matrix $\mathrm{B}$ on plant height is not so obvious.

In all, for the impact of seeding exercise matrix on the survival rate and growth situation of tissue culture seeding (including the plant height, growth situation and bud growth rate), the seeding exercise matrix prepared by fern root is most efficient. The survival rate, plant height, growth rate and bud growth rate have achieved $100 \%$, $4.5 \mathrm{~cm}, 43.67 \%$ and $54.33 \%$ respectively.

\section{Brief summary and discussion}

The demand for Dendrobium officinale Kimura et Migo in the market is increased year by year, but the planting technology has not kept pace with it, especially for the problems in survival rate of transplanting the tissue culture seeding of Dendrobium officinale Kimura et Migo . As the horny layer is not formed in the leaves before taking the tissue culture seeding of Dendrobium officinale Kimura et Migo out of the bottle, and it cannot carry out efficient photosynthesis. Seeding exercise is for transition of tissue culture seeding from enclosed and steady environment to a open and changeable one, gradually adapting itself to the natural environment. The Dendrobium officinale Kimura et Migo has a high demand on planting matrix, while its tissue culture seeding has much more demands on planting matrix. The completion of seeding exercise for tissue culture seeding shall take the facilitation of production increase for postplantation as the completion index. While this test mainly involves documentation and analysis on the survival rate and growth situation of tissue culture seeding (including plant height, growth rate and bud growth rate), which are both related to production. The test result indicates that, the impact of different shows matrix on survival rate of tissue culture seeding of Dendrobium officinale Kimura et Migo is different. In 5 matrix, the survival rate and 
growth situation of seeding exercise matrix $\mathrm{C}$ is the best, and its survival rate, plant height, growth rate and bud growth rate have achieved $100 \%, 4.5 \mathrm{~cm}, 43.67 \%$ and $54.33 \%$ respectively. This matrix is loose and has a good water permeability, proper for the root growth of Dendrobium officinale Kimura et Migo. Followed by seeding exercise matrix D, E and A. While the matrix of seeding exercise matrix B is hard and not easy to be crashed, thus improper for root intergrowth of Dendrobium officinale Kimura et Migo. Dendrobium officinale Kimura et Migo seeding has a high deman on growth matrix, and the tissue culture seeding newly out of the bottle is very fragile, while the matrix of Pine sawdust and fern root has a certain impact on the growth of Dendrobium officinale Kimura et Migo seeding. Pteridophyta grow in a wet and dark environment, one of the plants where most orchidaceae like to intergrow. The Dendrobium officinale Kimura et Migo tends to intergrow and its root can well absorb the gas-state moisture and oxygen in the air, providing a certain amount of nutrients for Dendrobium officinale Kimura et Migo. Pine sawdust is the matrix most Dendrobium officinale Kimura et Migo planter used for water conservation, and the its full mixture with fern root will help the root of Dendrobium officinale Kimura et Migo to better grow and absorb sufficient moisture. The matrix of fern root and pine sawdust are easy to be found in Guangxi, proper for growth of Dendrobium officinale Kimura et Migo. The current result is just for research of survival rate and growth situation, and it is required for further study on productivity. It is believed that if there's a decent increase in the productivity, the seeding exercise matrix will be worthwhile for numerous planters and farmers.

\section{Acknowledgement}

This paper is supported by the Science \& Technology Research Project of Colleges and Universities in Guangxi Province (GN: 2013YB318).

\section{References}

1. Li Shizhen. Compendium of Materia Medica [M]. Beijing: Beijing Science and Technology Press. 2006: 212.

2. Yan Zhaoxia. Ten Functions of "Life-saving Dianthera" of Dendrobium Candidum [Online]. Available from:http://health.ifeng.com/news/detail_2011_06/29 /7319646_0.shtml, in June 2011 .

3. Zhao Yongling, Wang Shilin \& Li Xiaoyu. Study on Dendrobium Aphyllum Polysaccharide [J]. Yunnan Plant Research. 1994, 16 (4):392-396.

4. Tian Yuanxiang. The Yellow Emperor's Canon of Internal Medicine Health Wisdom Book [M]. Shanghai: Shanghai Popular Science Press. 2011:41.

5. Ji Zhanhe. Preliminary research on China Dendrobium [J]. Journal of Plant Classification. 1980, 18(4): $427+449$.

6. Junlang Deng, Zheng Kuanyu et al. Problems and countermeasures existing in Yunnan medicinal Dendrobium industrialization [J]. Yunnan Agricultural Science and Technology, 2005:4.

7. Xie Fengxun, Practical Technology of Chinese Herbal Medicine Planting [M]. Beijing: Agriculture Press. 2001:406.

8. Chen Lianqing \& Pei Chi Tatsu this. Study on growth characteristics of Dendrobium mycorrhizal fungi in liquid culture [J]. Forestry Scientific Research. 2002, 15 (2):207-211.

9. Chen Lianqing, Pei Chi Tatsu this. Structure of three species of Dendrobium Mycorrhizal morphology and element component research [J]. Journal of Forestry Research. (1)96:100.

10. Wang Kangzheng. The cultivation of medicinal dendrobium research [J]. China Journal of Chinese Materia Medica.1998, 23 (6):340-343.

11. Wang Baoshan, The Test Statistic Method [M]. Beijing: China Agriculture Press. 2008:104. 